Ord. GRALLATORES.

Fam. Rallidae.

851. 7 Rallina tricolor Gr. 3 §, 3, 1 Skelett.

Fam. Oedicnemidae.

868. 2 Orthorhamphus magnirostris (Geoffr.). đ, $q$.

Fam. Charadriidae.

870. 1 Charadrius fulvus $\mathrm{Gm}$. ठ.

872.* 1 Aegialitis geoffroyi (Wagl.). ‥

873.* 1 " mongolica (Pall.). ㅇ.

Fam. Scolopacidae.

883. 3 Tringoides hypoleucus (L.). 3 ठ.

892. 4 Numenius variegatus (Scop.). 4 ․

Fam. Ardeidae.

897* 4 Ardea sumatrana Raffl. 2 đั ad., 1 đ juv., 1 q.

901. 8 Demiegretta sacra (Gm.). 4 ð, 2 o ad. normal, 1 o ad. (var. tota alba), 1 q juv.

907. 2 Butorides javanica Horsf. §’, ㅇ.

913. 2 Nycticorax caledonicus (Gm.). 2 ठิ.

Fam. Ibidae.

915.* 3 Ibis molucca (Cuv.). 1 o ad., 1 के juv., 1 ㅇ․ Ord. NATATORES.

Fam. Laridae.

936. 2 Sterna bergï Licht. 2 ठ.

In der Sammlung befinden sich mitbin 103 Species, von welchen Salvadori in seiner Ornithologia della Papuasia e della Molucche nur 81 auf Waigiu vorkommend aufführt. Alle mit einem * versehenen Nummern sind entweder neu oder wenigstens von Salvadori nicht ausdrücklich für Waigiu genannt.

\title{
Ornithologische Notizen aus Central-Afrika III.
}

$$
\text { Von }
$$

Dr. R. Böhm.

Mitglied der deutschen Expedition nach Ost-Afrika.

(Siehe Jahrg. 1883, Seite 162-208.)

An Herrn Herman Schalow.

Am 16. August hat eine durch einen Grasbrand entstandene Feuersbrunst unser Lager Waidmannsheil am Ugallafluss vernichtet. 
Ich habe dabei Alles verloren, was ich besass, mit Ausnahme des Wenigen, was ich auf dem Leibe hatte, und einiger Gewehre, die ich aus unserem mit Pulver- und Munitionsvorräthen angefüllten Hause reissen konnte. Meine beste Büchsflinte ist mit ziemlich sämmtlicher Munition der für Vogeljagd brauchbaren Hinterladergewehre vernichtet. Verbrannt sind nicht nur meine ganze persönliche Ausrüstung, nicht nur fast alles Präparir- und Conservirmaterial, nicht nur alle meine Bücher und Excerpte, sondern auch die seit Absendung meiner letzten Collection im Mai zusammengebrachten Sammlungen, dabei u. a. auch schöne oologische Präparate, meine gesammten Aufzeichnungen, meine in Europa angefertigten Zusammenstellungen über die Wirbelthierfaunen von Ostund Westafrika, alle meine Tagebücher, alle meine zoologischen, speciell ornithologischen Journale, meine botanischen Notizen, ferner alle meine Aquarellskizzen, mehr als 50 Blatt, die später auch zur Erläuterung landschaftlich-ornithologischer Darstellungen dienen sollten, endlich ausser einem ichthyologischen auch zwei soeben fertig gestellte ornithologische Berichte.

Der eine gab eine tabellarisch geordnete Uebersicht meiner in Uniamuesi gesammelten Beobachtungen über Zug, Brut und Federwechsel der Vögel nebst einer allgemeinen Einleitung über den Zusammenhang dieser mit den klimatisch-meteorologischen Verhältnissen des Jahres, der andere eine weitere Fortsetzung der ornithologischen Notizen.

Ersterer ist mit dem betreffenden Manuscript ganz verloren, letzteren versuche ich in Folgendem, soweit mir dies eben allein aus dem Gedächtniss möglich, zu reproduciren.

Da ich in diesem Jahre so wie so durch häufige und schwere Fieber, wie durch allerhand meiner speciellen Thätigkeit hinderliche Umstände - so war ich z. B. erst vor zwei Tagen von einer nothwendigen Reise nach Urambo zum Fluss zurückgekehrt, welche meine Zeit über einen Monat vollkommen anderweitig in Anspruch genommen hatte - sehr viel weniger zum Sammeln und Beobachten gekommen bin, als ich gewünscht hätte, so können die folgenden Notizen nur äusserst dürftig ausfallen.

Auch die nächste Zeit muss ich nun, statt, wie beabsichtigt, mit Sammeln am Ugallaflusse, mit Schreiben und neuen Zurüstungen, soweit diese eben thunlich, in Gonda zubringen. Ich habe jetzt nur noch die Hoffnung, dass meine abgeschickten Sammlungen und Berichte glücklich daheim anlangen, sonst ist meine bis- 
herige Thätigkeit in Afrika zum grössten Theil vergebens gewesen.

Das Unglück hat mich betroffen, während wir im Begriff stehen,' nach den Ländern zwischen Tanganika und Moëro-See, also einer ornithologischen, wie überhaupt naturwissenschaftlichen Terra incognita, abzugehen. Ich will dort versuchen, wenigstens ornithologisch zu sammeln, soviel ich kann, muss aber unter den vorwaltenden, unglücklichen Umständen um Nachsicht bitten, wenn meine Bemühungen ein mehr als unbefriedigendes Resultat liefern sollten.

Gonda im August 1882.

Dr. R. Böhm.

Graculus africanus.

Ende März langte ein Flug dieses Cormorans auf dem Ugallaflusse bei Waidmannsheil an, welcher wahrscheinlich wieder auf dem alten Nistbaum daselbst brüten wollte. Letzterer stand aber diesmal im Trockenen und strichen die Vögel längere Zeit, augenscheinlich unruhig und suchend, den Fluss auf und ab, ohne nachher daselbst zu nisten.

Im Juli sah ich mehrere Exemplare mit weisser Unterseite, leider fehlten mir aber Zeit und Gelegenheit, dieselben zu erlegen und ihr Geschlecht zu bestimmen. Da sich 1881 bestimmt kein solches Exemplar am Nistbaum befand, so dürften vielleicht nach beendeter Mauser die + 우 das halbweisse Kleid erhalten, die $\delta \delta$ dagegen das einfarbig dunkle beständig tragen.

Plotus Levaillanti.

Im März zur Paarungszeit sieht man diese Vögel, paarweise oder zu kleinen Trupps vereinigt, Flugkünste ausführen, welche man von diesen Vögeìn nie erwartet hätte. In schönen, ruhigen Spiralen kreisen sie gleich Raubvögeln hoch in der Luft umher oder fliegen reissenden Fluges geradeaus. Leider gelang es trotz vielfachen Umhersuchens nicht, die Nester zu entdecken, und werden die Vögel wahrscheinlich gemeinschaftlich am unteren Ugalla horsten. Herr Reichard beobachtete einmal einen Schlangenhalsvogel, welcher seinen Standpunkt auf dem Rücken eines schlafenden Flusspferdes genommen hatte.

Larus phaeocephalus Aud.?

Im Juli ein vereinzeltes Exemplar auf dem oberen Ugalla beobachtet. 
*314. Podiceps minor.

$\delta$ und $q$ von einem Eingeborenen auf einem kleinen Teiche bei Gonda gefangen (Bälge verbrannt).

*315. Thalassornis leuconota.

Diese eigenthümliche, kleine Ente fanden wir im März zu zweien (nicht paarweise) im Ugallaflusse auf einer von Pflanzen ganz bedeckten Strecke still im Wasser liegen. Aufgestört pflegten sie erst eine Strecke weit mit Hülfe der Flügel über das Wasser zu laufen, um dann schnurrenden Fluges dicht über die Wasserfläche hinzustreichen, wobei sie die Ständer in sonderbarer Weise steif nach hinten ausstreckten. Diese Enten liegen sehr fest und tief im Wasser und lassen sich ohne Mühe mit dem Kahne anfahren. Hier auch ein Flug von Dendrocygna viduata.

\section{Nettapus auritus.}

Im März paarweis und mit angeschwollenen Fortpflanzungsorganen,

Plectropterus gambensis.

Junge, welche noch halb mit Dunen bekleidet waren und sehr behend tauchten, wurden im Juli beobachtet. Die alte Gans sucht den Jäger von dem Aufenthaltsorte ihrer noch nicht flüggen Jungen fortzulocken, indem sie, ihre sonstige Scheu und Vorsicht ganz vergessend, mit ängstlichem Gepfeif dicht um ihn umherfliegt, auf dem Lande einfällt und hier, wie flügellahm, vor ihm herläuft.

\section{Porphyrio Alleni.}

Ein Exemplar hielt sich ganz vereinzelt bei Waidmannsheil an einer eng begrenzten Stelle einer mit Wasserpflanzen bedeckten Flussenge auf, war von uns dort bereits im März d. J. beobachtet worden und wurde dann im Juli erlegt (Balg verbrannt). Wahrscheinlich kommt die Art zusammen mit $P$. smaragdonotus häufiger am unteren Ugalla vor.

Ortygometra nigra.

Bei Tage sieht man diese Wasserhühner niemals, wohl aber bei Nacht im Mondschein auf dem offenen Wasser umherschwimmen.

Parra africana.

Die prachtvollen Eier im März und Juli erhalten.

Rhynchaea capensis.

Junges im Dunenkleid im Mai erhalten.

Ibis Hagedash.

Der Hagedash-Ibis ist hier nur am Wasser, nicht aber auch 
in der trockenen Savanne zu finden (cf. dag. F. u. H.). Am Ugallaflusse fast immer nur paarweis. (Balg verbrannt.)

Anastomus lamelliger.

Der Klaffschnabel kommt zur Regenzeit in grossen Schaaren in die überschwemmten Felder unmittelbar bei Gonda.

Ciconia Ab̈dimii.

Desgleichen. Zuweilen grosse Flüge vorüberziehend. Wahrscheiulich ist es diese Art, welche Dr. Kaiser einmal in Kakoma in so ungeheuren Massen überstreichend sah, dass der ganze sichtbare Himmel längere Zeit von ihnen erfüllt war.

Ciconia alba.

Zur Regenzeit, oft zusammen mit C. Abdimï, in den Feldern bei Gonda, wo die Störche Abends truppweis, zuweilen vermischt mit Ardea alba auf trockenen, einzeln im Felde stehenden Bäumen zur Nachtruhe aufhaken. Jedenfalls sind nicht alle derselben europäische Wintergäste, da ich sie noch Ende Mai beobachtet habe. Nach der Regenzeit verstreichen sie sämmtlich.

Ardea minuta.

Ein einzelnes Exemplar Abends in einem Sumpf bei Gonda beobachtet.

Ardetta Sturmii.

Im Mai zwei weisse Eier mit dem auf dem Neste gefangenen q erhalten.

Lobivanellus lateralis.

In kleinen Trupps ständig bei Gonda und Urambo.

Francolinus rubricollis.

Ein frisches Gelege noch im Juni erhalten, während sonst zu dieser Zeit die Jungen sämmtlich flügge waren. Morgens gehen die Ketten dieses Francolins, umherlaufend, suchend und kratzend, ihrer Nahrung nach, gegen Mittag liegen sie dann ziemlich fest unter Büschen, namentlich gern an und auf alten Termitenhügeln. Zuweilen findet man die Vögel auch am Tage aufgebäumt, und hört man dann von ihnen zuweilen ein sonderbares, klägliches Pfeifen. Aufgescheucht rufen sie laut und abgebrochen und erheben, wenn sie sich wieder gesammelt, ein sonderbares, gellendes, abgebrochenes Geschrei. Dieses Geschrei wird mit sinkender Sonne, zu welcher Zeit die Ketten wieder rege werden, mehr und mehr laut. Abends ziehen sie dann entweder zum Wasser oder in die offene boga hinaus, wobei die Henne mit ihren Jungen häufig aufsteht und lärmend ins Freie streicht. Der alte Hahn hält sich 
gern etwas abseits und bäumt, zuweilen in beträchtlicher Höhe, in der Nähe auf. Auf seinem Sitze hält er sich sehr ruhig, höchstens schüttelt er mal das Gefieder. Sobald aber der Ruf eines anderen Hahnes herüberschallt, erhebt er auch, heftig mit Kopf und Hals nickend, sein schmetterndes „Körreck, körreck“. Endlich streicht er laut lockend seiner vorangezogenen Kette nach.

Francolinus sp.

(Die kleine Art, vielleicht Schlegelii. ?)

Mehrmals 3 und 6 Eier erhalten. Die eigenthümlichen, hellklingenden, dem einer Kindertrompete nicht unähnlichen Stimmlaute dieses kleinen Wald-Francolins hört man besonders gegen Abend durch den puri schallen. Den zweisilbigen, oft wiederholten Ruf des Hahns übersetzen die Waswaheli mit "Qua-upe, Qua-upe“ (nichts da!) und soll derselbe den Jäger von der Suche abrathen, weil kein Wild in der Nähe sei. Am 5. August eine Kette mit flüggen Jungen beobachtet. Solche Ketten entschliessen sich oft nur sehr schwer zum Aufstehen, die Vögel bleiben nach kurzem Lauf mit hochgerecktem Hals stehen, entfernen sich auch nicht weit von einander, so dass man mehrere hintereinander erlegen kann.

Numida coronata.

Gelege vom Februar bis Mai erhalten. Junge im Flaumkleid (zu welchen auch der Francolinus pull.? der ersten Sendung gehört) im März, flügge im Juni erhalten, von welcher Zeit an man auch häufig solche bei den Ketten sah; Junge mit noch ungefärbten, schwärzlichen Nackttheilen am Kopfe am 18. September erhalten. (Bälge juv. u. ad. verbrannt.)

\section{Helotarsus ecaudatus.}

Ein ausgefärbtes Exemplar wurde am Ugallaflusse von einem Eingeborenen beim Kröpfen, angeblich von Fischen, wohl aber von einem Varanus oder dergl. mit den Händen gefangen. Bei der Missionsstation in Urambo hat ein Pärchen auf einem hohen Baum gehorstet und die zwei im Horst aufgefundenen Eier auch glïcklich ausgebracht. Nähere Daten habe ich nicht in Erfahrung gebracht.

*316. Vultur occipitalis.

Ein ơ unter einer Schaar von Gyps leuconotus und Neophron pileatus bei dem Cadaver eines am Abend vorher geschossenen Zebras erlegt. Die nackten Kropf- und Halstheile im Effect hochroth gefärbt. (Bälge von Vult. occ. u. Gyps leucon. verbrannt.) 
Oena capensis.

Ende März 2 bereits stark bebrütete Eier dieser kleinen langschwänzigen sp. in einem flachen, losen Neste am Ugallaflusse gefunden.

Picus sp. (mit Braun an Kinn und Kehle).

Stösst einen hellen, quiekenden Ruf aus. Im April sehr verblichen.

Centropus superciliosus.

Im Mai (?) erhielt ich die drei reinweissen Eier dieses Kukuks. Das colossale, in einer Bananenstaude dicht bei Gonda angelegte Nest war unordentlich aus Bananenblättern und Rispen zusammengefügt, von welchen die letzteren vorzuiglich die Basis bildeten. Höhe von 25, Durchmesser von $30 \mathrm{~cm}$. Die Nistmulde war nur flach, verhältnissmässig klein und ohne jede Auspolsterung. Auffallender Weise erhielt ich zweimal von verschiedenen Personen hellbräunliche, spärlich rothbraun gefleckte Eier (Gel. No. ?), welche mit Bestimmtheit dem „Widi-Widi“ zugeschrieben wurden und auf der Erde im Grase gefunden sein sollten. Strophe eines PagaziGesangs: „O! widi-widi walakala“, „Ho! der Widi-Widi ist hingefallen!“ (Spott über ermüdete, Rast machende Genossen.)

Oxylophus afer.

Im März auffallend häufig am Wala. đo im Uebergangskleid im Juli. Im Allgemeinen gleich der betreffenden Beschreibung von v. Hgl., aber Kropf und Brust mehr isabellgelb wie rauchgrau, die schwärzliche Strichelung bedeckt Kehle und Halsseiten; der weisse Spiegel auf dem Flügel bereits entwickelt. Schwanz oben schwärzlich mit blauem und grünem Metallglanz, äusserste Steuerfeder an der Aussenseite mit weissem Längsfleck, der nicht bis zur Spitze geht und bei der rechts bereits in der Mitte schmal beginnt.

*317. Coccystes glandarius.

Wahrscheinlich war es diese Art, welche am Wala ihr durchdringendes: „Rück, rück“ bis tief in die Nacht hören liess. Auf Viehweiden bei Gonda habe ich den Häherkukuk mehrfach bemerkt, ein Exemplar (verbrannt) wurde im Orte selbst erlegt.

Chrysococcyx cupreus.

Im April hörte man häufig die laute, raubvogelartige Stimme der $ઠ ð$ von den Mrumbabäumen im Orte Gonda selbst erschallen. Die erlegten Exemplare waren sämmtlich đ̛đ̛. Bei Kakoma habe ich den Goldkukuk nie bemerkt. 
Trachyphonus sp.

Das laute, gleich dem Schlagen einer losgehenden Weckeruhr losgehende Zetern dieses eigenthümlichen Vogels schlägt zuweilen in ein sanftes, nicht unangenehm klingendes, auf- und absteigendes Trillern um, was einen ganz sonderbaren Effect macht.

Corythaix chlorochlamys.

Ruft dieser Vogel nur dreimal hintereinander sein „kuck kuck kuck", so gilt dies bei den Waniamuesi für ein derartiges schlechtes Vorzeichen, dass sogar ganze Karavanen auf ihrem Wege deshalb Halt machen.

*318. Geocoraphus sp. (sehr ähnlich modestus v. Hgl.).

Im Juni auf einer von Wildsauen tief aufgebrochenen Sandbank am Ugalla geschossen. (Balg verbrannt.)

Coraphites sp.?

Soll den Rinderheerden folgen und sich auf dem Rücken der Thiere niederlassen.

Crithagra butyracea.

In grosser Zahl in den verwüsteten, öden Waldrändern des puri längs der Feldlichtungen von Gonda. Im Januar ein $q$ mit ausgebildetem, doch noch schalenlosem Ei im Legeschlauch geschossen.

Passer Swainsoni.

Mehrfach Gelege und Junge erhalten.

Die verhältnissmässig grossen, unordentlich aus Stroh und Hühnerfedern zusammengefügten Nester fand ich an den verschiedensten Oertlichkeiten, so in Mauerlöchern, in den Astgabeln von Bäumen in der Nähe der Orte, einmal auch im Blattquirl eines starken Schilfstengels im Sumpfe dicht bei Gonda.

Habropygia astrild.

Junge dieser sp. (?): mit Roth an der Schwanzbasis. Mundwinkel weiss, an den Ecken leuchtend ultramarinblau.

Uraeginthus phoenicotis.

Noch im Juni ein frisches Gelege gefunden. Die unordentlich aus feinen Grasrispen und einigen weichen Numida- oder Francolinus-Federn gleichsam zusammengeballten Nester stehen im Gestrüpp zuweilen unmittelbar über dem Erdboden.

Penthetria eques.

Im Februar und März sah ich häufig Trupps dieser hübschen Wittwe, gewöhnlich aus einem $\delta$ und mehreren $q$ 을 bestehend, auf Waldwiesen um Gonda. Da ich zur Zeit gewöhnlich durch an- 
dauernde Fieber ausser Stand gesetzt war, etwas zu unternehmen, gelang es mir nicht, einen der sehr scheuen Vögel zu erlangen. Im Mai waren sie sämmtlich verschwunden, während sich von $P$. macroura noch einzelne Exemplare zeigten.

Hyphantornis nigriceps?

Auf einer hohen, für heilig und deshalb unberührbar geltenden, von uns indess im oologischen Interesse nicht respectirten Sycomore im Orte Gonda befindet sich eine ständige Brutcolonie. Im Februar waren die Vögel eifrig beschäftigt, die alten Nester auszubessern oder neue anzulegen. Sämmtliche waren derartig an den äussersten Zweigspitzen befestigt, dass es nicht möglich war, intacte Gelege $\mathrm{zu}$ erhalten.

Am 18. Februar enthielt ein Theil der Nester bereits Eier, im März trugen die Vögel aber noch immer, mit ungeheurem Gelärm und Geschrei ab- und zufliegend, Nistmaterial aus dem Sumpfe vor dem Thore, in dessen Schilfmassen sie auch zu übernachten pflegten.

Mitte März gelangten wir, den Wala hinabfahrend, in den Ugallafluss und fanden hier mehrere der dichten, über das Wasser selbst hängenden und von einer charakteristischen Baumart (Vergl. Herb. No. 81 ?) gebildeten Gebüsche mit Brutcolonien dieses Webers besetzt.

An einem derselben 'waren die Vögel noch bis Anfang April mit Bauen beschäftigt, zwei andere wurden von uns am 29. und 30. März ausgebeutet. Das volle Gelege besteht aus drei Eiern, welche theils einfarbig grünblau, theils mit rostbraunen Fleckchen bedeckt sind. Zuweilen findet man solche, die nur sehr spärlich gefleckt sind, also gleichsam Uebergangsformen, nie aber einfarbige und gefleckte in demselben Nest. Die aus grobem und flachem Material geflochtenen und zuweilen mit Mimosenblättern ausgefütterten Nester waren auch hier an den äussersten Zweigenden über dem Wasser befestigt und hatten dann und wann eine enge, verlängerte Eingangsröhre. Später fanden wir die Colonien durchaus verlassen.

*319. Hyphantornis olivacea.

Dieser schöne Weber ist ein einsam und ungesellig lebender Vogel, der seine Nester nur an den Flüssen, am liebsten in im Wasser selbst stehenden Büschen anlegt. Jedes Paar hat hier sein eigenes, ausgedehntes Revier, in welchem es immer wieder denselben Nistbusch benutzt, indess jedes Jahr neue Nester baut, und 
zwar, wie es seheint, stets ein Brut- und ein kleineres Spielnest. Die rundlichen, fest gebauten Nester werden an den äussersten Enden langer, schwankender und möglichst unerreichbarer Zweige befestigt, hängen aber bei hohem Wasserstande zuweilen bis dicht über dem Wasser herab.

Wie andere Weber beisst auch diese Art alle Nebenästchen und Blätter dieser Zweige sorgfältig ab. Beim Bau wird mit der kranzförmigen Thür begonnen, welche anfangs schräg nach oben gerichtet ist und erst später, wenn sich der Zweig unter der Last des Nestes bogig gesenkt hat, nach unten zu liegen kommt.

Das Gelege besteht nur aus zwei Eiern, welche sehr verschieden gefärbt sind, entweder einfarbig grünlichblau oder auf bläulichem oder weisslichem Grunde rothbraun gefleckt. Einmal fand ich ein einzelnes, auffallend längliches und lebhaft grünblau gefärbtes Ei in einem Neste. Ende März fand ich stark bebrütete Eier und.am 23. (?) zwei Junge, ein nacktes und ein bereits befiedertes. Die alten Vögel sind ausserordentlich scheu, kommen selten aus dem dichtesten Laubwerk der Uferbäume und Büsche hervor und kehren, wenn man ein Nest untersucht, erst nach langer Zeit und wenn man vollkommen gedeckt steht, zu demselben zurück.

*320. Lanius minor Gml. (?).

Ein Exemplar (Balg verbrannt) Anfang Mai im Gebüsch einer Viehweide bei Gonda erlegt.

Dryoscopus hamatus?

ㅇ im März mit hirsekorngrossen, gelben Eiern am Ovar.

Rhynchastatus funebris.

Im März ein Gelege der röthlichbraunen, an die Farbe gewisser Stachelbeeren erinnernden Eier in einem flachen Neste im Gebüsch gefunden.

Muscicapa grisola.

Im März noch am Ugallaflusse gefunden.

Dicrurus divaricatus.

Stösst über Gewässern nach Insekten jagend, bis auf den Spiegel selbst herab.

Campephaga nigra.

Im Juni ơ im Uebergangskleid: Oberseite blauschwarz, überall mit graubraunen Eedern vermischt, Unterseite weiss, jede Feder mit schwarzer Querbinde vor dem Ende, überall mit stahlblauschwarzen Federn untermischt, Kinn und Kehle ganz stahlblau- 
schwarz. Kleine Deckfedern und grosse Deckfedern der Schwingen 2. Ordnung breit gelb gerandet, Schwingen hellgelb mit schwärzlichem Längsfleck, letzte Schwingen gleich den Deckfedern. Ohrgegend weiss und blauschwarz, Tibialbefiederung gelb und blauschwarz gemischt.

\section{Pycnonoțus nigricans.}

Im März am Wala das runde, dünnwandige, ziemlich tiefmuldige und mit zwei nackten Jungen besetzte Nest in einem ziemlich spärlich belaubten, im Wasser selbst stehenden Busch in ca. $3 / 4$ Mannshöhe gefunden.

Crateropus sp. (gleich denen vom Wualaba).

Im Januar ein vereinzeltes ${ }^{\star}$ mit kolossal geschwollenen Hoden bei Gonda erlegt.

\section{Crateropus Jardinei?}

Im März und April erhielt ich mehrere Gelege. Auffallend ist mir, dass ich sowohl von Eingeborenen das o dieser kleinen sp. mit den Eiern, auf dem Neste gefangen, erhielt, als selbst beim Neste erlegte, während ich sonst stets nur den sehr gemeinen Crateropus kirki (?) schoss. Ein im April geschossenes Exemplar dieser letzteren sp. befand sich jedenfalls nicht in der Fortpflanzungszeit und mauserte die Schwungfedern.

\section{Molacilla alba.}

Die sehr lange Fortpflanzungsperiode aus den eingeschickten Gelegen zu ersehen. Die Kehlfärbung ist zu keiner Zeit ganz schwarz.

*321. Camaroptera?

\section{(Bälge verbrannt.)}

Dieser kleine Vogel lebt äusserst still und versteckt im tiefsten Dickicht dunkler, verworrener Büsche, wo er zaunkönigartig einen leisen, eintönigen, kläglichen Laut ausstossend, umherschlüpft. Im März fanden sich Gesellschaften von Alten mit mehreren Jungen vor. Von dem đo glaube ich einen schnalzenden Gesang gehört zu haben.

đ Oberseite olivengrün, Flügel olivengrün, Schulterfleck gelb, Unterseite weiss, Kehle und Brust hellgelb, letztere mit schwärzlichem Stern; Iris rehbraun, Füsse fleischfarben, Schnabel schwarz.

juv. (et ? ?): Unterseite ganz weiss.

pull.: dto., das Olivengrün weniger lebhaft, die nackten Mundwinkel weisslich, Füsse heller, Iris dunkelbraun. 


\section{Acrocephalus sp.}

Diese Rohrdrossel hält sich zur Regenzeit in den dichten, mit riesigem Schilf, Bananen und undurchdringlichem Gestrüpp untermischten und zum Theil unter Wasser gesetzten Euphorbienhecken rings um Gonda auf. Von hier lässt sie ununterbrochen ihr anheimelndes Geknarr hören, zeigt sich aber nur selten und auf flüchtige Augenblicke. An derselben Localität häufig Calamoherpe, Pycnonotus, Pyromelana, Dicrurus, Drymoeca, Centropus, Ispidina picta.

*322. Calamoherpe sp.

An derselben Stelle wie die vorige sp. und stets im Innern der Gebüsche, daher die aus nächster Nähe geschossenen Exemplare sehr zerstört. Der leise fortschwatzende Gesang gleicht ganz dem unserer Calamoherpen.

Dry moeca sp.

No. 220.

Im April die nackten Jungen erhalten. Dieselben sehen ausserordentlich sonderbar aus, indem sie mit hellrehbraunem Flaum bedeckt sind, welcher auf dem Kopf eine Krone, wie die eines Pfauenkranichs bildet, und der Rachen inwendig von schneeweisser Farbe ist, von welcher sich tiefschwarze Flecken abheben.

Nectarinia collaris.

Im März mit angeschwollenen Sexualorganen.

Nectarinia gutturalis.

Kommt nicht nur in den Bananenanpflanzungen bei den Ortschaften, sondern auch, in Gemeinschaft mit anderen Nectarinien, auf den sonnigen, bebuschten Hängen der Granitkuppen, welche hier und da aus dem bebauten Land rings um Gonda aufragen, und selbst, fern von menschlichen Niederlassungen, im Ufergebüsch des Ugallaflusses vor. Im . . . . . e erhielt ich ein schönes Nest dieser sp. mit vorspringendem Schutzdach über dem Eingang (eingesandt). Die Eier waren vom Finder leider zerbrochen.

Irrisor erythrorhynchus.

Ein leicht angeschossenes Exemplar versuchte sich in ein Mauseloch zu verkriechen, in dem es jedoch seinen langen Schwanz nicht unterzubringen vermochte, wusste sich, nach Haus gebracht, mit grosser Geschicklichkeit seiner Fussfessel zu entledigen und kletterte schnell und gewandt die Hauswand hinauf.

Merops apiaster.

Noch im März bei Gonda auf dem Durchzuge. 


\section{Merops cyanostictus.}

juv.: Das Kehlband durchaus fehlend, der hellblaue Superciliarstreif dagegen deutlich und breit. Iris rehbraun.

\section{Ceryle maxima.}

Der Flug dieses, auch in diesem Jahre in einigen Exemplaren am Ugallafluss beobachteten, grossen Eisvogels ist auffallend still und weich.

Obgleich das erlegte $q$ (Balg verbrannt!) einen Schuss mit Entenschrot erhalten, versuchte es doch mit Energie schwimmend zu entkommen.

\section{Halcyon senegalensis.}

Ausserordentlich häufig und gar nicht scheu auf dem Walaflusse. Im März hier stets gepaart und am ...... zwei aus einem Baumloche genommene Junge erhalten.

Hirundo senegalensis.

Die Senegalschwalbe nistet in der That in Baumlöchern. Wir fanden das Nest im März in einem hohlen, aus dem Wasser selbst ragenden Baumstamm. Die Höhlung war ca. 1 Meter tief und die Eingangsöffnung bis auf ein kleines Flugloch mit Lehm sehr fest vermauert. Ein einziges weisses Ei mit bereits ziemlich entwickeltem Embryo lag im Grunde auf einer Unterlage von etwas Stroh und Federn, wurde jedoch leider durch herabfallende Lehmstücke während der bei der starken Strömung sehr mühsamen Erweiterung der Oeffnung vom Boot aus zerbrochen. Um dieselbe Zeit sah ich auch diese Schwalbe Strohhalme aus den Dächern von Hütten reissen und mit diesen, welche sie jedenfalls zum Nisten benutzen wollten, umherfliegen.

Hirundo puella.

Diese schöne kleine Art ist in Uniamuesi wie am Zambesi Hausschwalbe. Ich fand ihr aus Lehm gebautes, halbkugliges, ziemlich dünnwandiges und mit einer grossen Eingangsöffnung versehenes Nest an der Innenseite des Strohdachs eines Schuppens auf der englischen Missionsstation in Urambo. Dasselbe enthielt in der ersten Hälfte Juli nackte Junge. Bei Kakoma und Gonda nie bemerkt.

\section{Caprimulgus sp.}

(Die bunte Art mit dem eigenthümlichen Ruf. Eine schöne Suite von Bälgen verbrannt.)

Dieser schöne Ziegenmelker ist am Ugallaflusse ausserordentlich häufig, wo er tagsüber unter Büschen, besonders längs des Fluss- 
ufers selbst, sehr fest liegt und, nachdem er sich, aufgescheucht, wieder niedergelassen, mit einiger Uebung ganz gut $\mathrm{zu}$ entdecken ist. Einigemal fand ich Pärchen dicht zusammen sitzend; die erlegten Exemplare waren auffallender Weise sämmtlich 우. Abends schweben diese und andere Ziegenmelker über Sandbänken und ganz kurzrasigen Stellen am Flusse Insekten haschend hin und her, setzen sich von Zeit zu Zeit und fliegen von ihrem Standpunkte, einem Insekte nach, leise weiche Laute ausstossend, auf und wieder zurück.

Beobachtet, aber trotz vieler Bemühungen nicht erlegt und sicher bestimmt, wurden von mir in Uniamuesi noch folgende, meist den Raptatores angehörige Arten:

1. Ardea ardesiaca.?

Cf. Ber. I.

2. Ardea sp.

Einmal sah ich, auf dem Ugalla kreuzend, eine kleine, sehr dunkle Art mit anscheinend braunrothen Schulterdecken vorüberfliegen.

3. Ardea sp.

Klein und sehr bunt gezeichnet, nur ein Exemplar gesehen. 4. Otis sp.

Mehrmals im Hochgras und in Feldern aufgestört. Cf. Ber. I.

5. Caccabis sp.

Tabora. cf. Ber. I.

6. Spizaëtos (bellicosus?).

Auffallend grosse und starke Art, unten im Ganzen weiss, mit schwarzer Kopfzeichnung. Einigemale im puri auf hohen Baumwipfeln und am Luderplatze bei Kakoma beobachtet.

7. Spizaëtos (occipitalis?).

Jedenfalls auf diese Art $\mathrm{zu}$ beziehen. Tief dunkelbraun mit weisser Flügelzeichnung und langer, schmaler Haube. Stimme hellblökend. Einigemale am Ugallaflusse gesehen.

8. Falco (biarmicus?).

Ziemlich sicher diese Art. Unterseite hellröthlichgelb, mit schwarzem Bartstreif. Bei Gonda und Sassagula in Ugalla.

9. Aquila (rapax?).

Mittelstarker, brauner Adler. Ziemlich selten.

10. Elanus melanopterus?

Einen kleinen grauweissen Falken, den ich einige Male auf 
Feldbäumen bei Gonda sah, glaube ich auf diese Art beziehen $\mathrm{zu}$ können. Ein junges, vielleicht $\mathrm{zu}$ dieser sp. gehöriges, von Herrn Reichard präparirtes Exemplar ist, ehe ich es näher untersuchen konnte, mit verbrannt.

\section{Buteo?}

Kräftige, anscheinend gebänderte Art.

12. Melieras sp.

Ein schönes, aber an Flügeln und Schwanz bis zur Unbrauchbarkeit verstümmeltes Exemplar nach Gonda von Eingeborenen gebracht.

13. Circus sp.

Braun, mit weissem Bürzel (!). Vereinzelt von Gonda bis zum Tanganika gesehen.

14. $B u b o$ sp.

Von der Grösse unseres B. maximus, anscheinend quer gebändert. Ein Exemplar im dichten puri bei Kakoma aufgestört, ein zweites einige Male in der Dunkelheit am Wualaba bemerkt, ohne schussrecht ankommen zu können.

15. Syrnium sp.?

Eine grössere, gebänderte Eule einmal bei Gonda zwischen Granitfelsen aufgestört. Auch Herr Reichard hat mehrmals grössere Eulen gesehen, von welchen eine aus einem alten Scopus Horst, den er zu ersteigen versuchte, herausflog.

16. Colius (striatus?).

Tanganika. Cf. Ber. II.

17. Barbatula sp.

In einem dichten Ufergebüsch am Ugallaflusse sah ich, durch einen eigenthïmlichen Stimmlaut aufmerksam gemacht, einen kleinen Bartvogel, der indess durch den Schuss aus nächster Nähe in Fetzen zerrissen wurde. Jedenfalls war er mit Gelb und Roth gezeichnet.

18. Hypochera nitens.?

Cf. Ber. II. Glaube ich auch einmal an der Tränke auf einer Sandbank am Ugalla gesehen zu haben.

19. Merops (nubicus?).

Mehrmals sahen wir einen Merops mit rother Unterseite hoch über dem Ugallaflusse vorüberfliegen. Offenbar befanden sich die Vögel nur auf dem Durchzuge. 

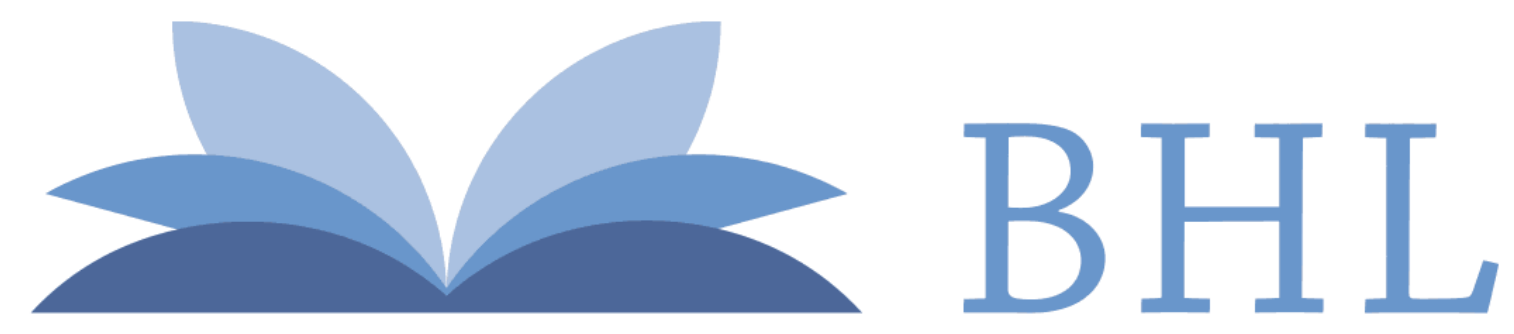

\section{Biodiversity Heritage Library}

1885. "Ornithologische Notizen aus Central-Afrika III." Journal fu

r Ornithologie 33, 35-49. https://doi.org/10.1007/bf02007337.

View This Item Online: https://www.biodiversitylibrary.org/item/101743

DOI: https://doi.org/10.1007/bf02007337

Permalink: https://www.biodiversitylibrary.org/partpdf/142323

\section{Holding Institution}

Smithsonian Libraries

\section{Sponsored by}

Biodiversity Heritage Library

\section{Copyright \& Reuse}

Copyright Status: Public domain. The BHL considers that this work is no longer under copyright protection.

This document was created from content at the Biodiversity Heritage Library, the world's largest open access digital library for biodiversity literature and archives. Visit BHL at https://www.biodiversitylibrary.org. 